\title{
BMJ Open Efficacy of neoadjuvant hyperthermic intraperitoneal chemotherapy in advanced high-grade serous ovarian cancer (the NHIPEC trial): study protocol for a randomised controlled trial
}

\author{
Miao-Fang Wu, ${ }^{1}$ Li-Juan Wang, ${ }^{1}$ Yan-Fang Ye, ${ }^{2}$ Chang-Hao Liu, ${ }^{1}$ Huai-Wu Lu, ${ }^{1}$ \\ Ting-Ting Yao, ${ }^{1}$ Bing-Zhong Zhang, ${ }^{1}$ Qing Chen, ${ }^{1} \mathrm{Ji}-\mathrm{Bin} \mathrm{Li}$ (D) ${ }^{3}$ Yong-Pai Peng, ${ }^{1}$ \\ Hui Zhou, ${ }^{1}$ Zhong-Qiu Lin, ${ }^{1}$ Jing Li (i) ${ }^{1,4}$
}

To cite: Wu M-F, Wang LJ, Ye Y-F, et al. Efficacy of neoadjuvant hyperthermic intraperitoneal chemotherapy in advanced high-grade serous ovarian cancer (the NHIPEC trial): study protocol for a randomised controlled trial. BMJ Open 2021;11:e046415. doi:10.1136/ bmjopen-2020-046415

- Prepublication history for this paper is available online To view these files, please visit the journal online (http://dx.doi. org/10.1136/bmjopen-2020046415).

Received 30 October 2020 Accepted 22 November 2021

Check for updates

(C) Author(s) (or their employer(s)) 2021. Re-use permitted under CC BY-NC. No commercial re-use. See rights and permissions. Published by BMJ.

For numbered affiliations see end of article.

Correspondence to

Dr Jing Li;

lijing228@mail.sysu.edu.cn

\section{ABSTRACT}

Background Neoadjuvant chemotherapy (NACT) is an important treatment option for patients with ovarian cancer. Although intravenous NACT can improve optimal resection rates and decrease surgical morbidity and mortality, these advantages do not translate into a survival benefit. Ovarian carcinoma is mainly confined to the peritoneal cavity, which makes it a potential target for hyperthermic intraperitoneal chemotherapy (HIPEC). Our previous study showed that HIPEC could be used in the neoadjuvant setting, which was named neoadjuvant HIPEC (NHIPEC). Since hyperthermia is an excellent chemosensitiser, we hypothesised that the combination of NHIPEC and intravenous NACT could show superior efficacy to intravenous NACT alone.

Methods This study is a single-centre, open-label, randomised (1:1 allocation ratio) phase 2 trial. $A$ total of 80 patients will be randomly assigned into an experimental group (NHIPEC+intravenous NACT) or a control group (intravenous NACT). Patients in the experimental group will receive NHIPEC following laparoscopic evaluation, and four tubes will be placed via the laparoscopic ports, which will be used to administer NHIPEC. Then, perfusion with docetaxel (60$\left.75 \mathrm{mg} / \mathrm{m}^{2}\right)$ will be performed $\left(43^{\circ} \mathrm{C}\right.$ for $60 \mathrm{~min}$, Day 0$)$ followed by cisplatin $\left(75 \mathrm{mg} / \mathrm{m}^{2}\right.$, Day 1$)$ infusion $\left(43^{\circ} \mathrm{C}\right.$ for 60 min) 24 hours later. After NHIPEC, two cycles of intravenous NACT will be given. Patients in the control group will receive three cycles of intravenous NACT. The primary endpoint is the proportion of patients who achieve a Chemotherapy Response Score (CRS) of 3 according to the CRS system. The secondary endpoints include progression-free survival, overall survival and the rates of complete resection and NHIPEC-related adverse events.

Ethics approval and dissemination This study was approved by the Ethics Committee of Sun Yat-sen Memorial Hospital (approval number: 2020-ky-050). Results will be submitted to peer-reviewed journals and presented at national and international conferences. Trial registration number ChiCTR2000038173.

\section{Strengths and limitations of this study}

- This is the first randomised trial that investigates the role of hyperthermic intraperitoneal chemotherapy (HIPEC) in the neoadjuvant setting.

- The Chemotherapy Response Score system is used as the primary outcome measure facilitating timely assessment of the efficacy of neoadjuvant HIPEC.

- A possible limitation is interoperator variations in terms of operational technique.

\section{INTRODUCTION}

For patients with newly diagnosed advanced epithelial ovarian cancer, surgical staging and maximal cytoreduction followed by platinumbased chemotherapy has been the cornerstone of treatment. ${ }^{12}$ There is convincing evidence that the amount of residual disease following cytoreductive surgery has a significant impact on patient prognosis. ${ }^{3}$ Since each $10 \%$ increase in optimal cytoreduction was associated with a 2.3-month increase in median survival, ${ }^{4}$ reduction to no visible disease (R0 resection) is the ultimate goal of surgery. However, to achieve this, high complexity surgeries are required in primary debulking surgery (PDS), which increases the risk of surgery-related morbidity and mortality. ${ }^{5-7}$ For patients with high perioperative risk and those with a low likelihood of achieving R0 resection in PDS, neoadjuvant chemotherapy (NACT) followed by interval debulking surgery (IDS) can be considered a reasonable alternative. Three phase III trials have compared NACT-IDS with PDS and showed survival non-inferiority of NACT-IDS. ${ }^{8-10}$ In addition, patients treated with intravenous NACT were less likely to suffer postoperative morbidity and mortality. ${ }^{89}$ The amount of 
residual disease after cytoreduction has been the strongest prognostic factor for patients with high-grade serous ovarian carcinoma (HGSOC). ${ }^{11}$ However, as demonstrated in the three randomised trials, NACT can increase the chances of achieving $\mathrm{R} 0$ resection, but it cannot offer better survival outcomes when compared with PDS. ${ }^{8-10}$ While this may be a result of high tumour burden or patients' poor performance status, poor response to intravenous NACT could be a possible reason. Since tumour response to NACT plays an independently prognostic role for patients with HGSOC and the efficacy of NACT and IDS is still a matter of debate in gynae-oncology oncology literature, ${ }^{12-15}$ we believe that enhancing the efficacy of NACT has the potential to improve survival outcomes.

For patients with ovarian cancer, the disease is mainly confined to the peritoneal cavity. Because of the plasmaperitoneal barrier, the peritoneum is poorly reached by traditional intravenous NACT. There is increasing evidence that hyperthermic intraperitoneal chemotherapy (HIPEC) is an effective treatment for peritoneal carcinomatosis. ${ }^{15-18}$ In a randomised controlled trial by van Driel et al, the addition of HIPEC to IDS resulted in longer recurrence-free survival and overall survival (OS) than surgery alone. ${ }^{19}$ A recent retrospective study conducted at five high-volume cancer centres in China investigated the efficacy of HIPEC in 584 patients with ovarian cancer. ${ }^{20}$ The authors reported that the median OS time was significantly longer for patients who underwent HIPEC following PDS than for those who underwent PDS alone (median OS: 49.8 months vs 34.0 months). Our previous study showed that HIPEC could be used in the neoadjuvant setting, which was named neoadjuvant HIPEC (NHIPEC). ${ }^{21}$ A total of 14 patients with a Fagotti score $\geq 8$ received NHIPEC followed by two cycles of intravenous NACT, and NHIPEC-related adverse events (AEs) of grade 4 were not observed. The most common events were neutropenia and abdominal pain. Following NHIPEC, all patients received two subsequent cycles of NACT without dose delay or dose reduction. These findings suggested that NHIPEC is a feasible option for patients with ovarian cancer with a low likelihood of achieving optimal cytoreduction during PDS. $^{21}$ Since hyperthermia is an excellent chemosensitiser and there is evidence that HIPEC is beneficial for selected patients with ovarian cancer, ${ }^{19} 2022{ }^{23}$ we hypothesised that the combination of NHIPEC and intravenous NACT could show superior efficacy to intravenous NACT alone.

The Chemotherapy Response Score (CRS) system was developed by Böhm et al for patients with HGSOC who were treated with NACT-IDS..$^{12}$ It is a three-tiered system based on the pathological examination of residual disease on omental specimens following NACT. Since its description, the CRS system has been independently assessed in many studies. ${ }^{13}{ }^{24-27}$ Recently, the HGSC CRS Collaborative Network validated the prognostic role of the CRS system in a real-world, heterogeneous study population. ${ }^{25}$ Since CRS 3 was independently associated with significantly improved prognosis, it has been proposed as a surrogate for both progression-free survival (PFS) and OS. The International Collaboration on Cancer Reporting (ICCR) guidelines and European Society for Medical Oncology guidelines also recommend the routine use of the CRS system for patients with HGSOC to evaluate the tumour response to NACT and predict prognosis. $^{2}{ }^{28}$ Given current evidence, we chose this system as the primary method for evaluating the efficacy of NHIPEC.

\section{METHODS AND ANALYSIS \\ Study design}

This is a single-centre, open-label, randomised (1:1 allocation ratio) phase 2 trial. The eligible patients will be randomised into an experimental group (NHIPEC +intravenous NACT) or a control group (intravenous NACT). Patients in the NHIPEC experimental group will receive NHIPEC and two cycles of intravenous NACT, while patients in the control group will receive three cycles of intravenous NACT. All patients will undergo IDS within 4 weeks after the last cycle of NACT. The specimens obtained during IDS will be formalin-fixed and paraffin-embedded. The chemotherapy response will be determined by three independent pathologists. The CRS score will be assigned based on omental examination as described by Böhm. ${ }^{12}$ Following IDS, all patients will receive at least three cycles of systemic platinum-based chemotherapy. ${ }^{1}$ Figure 1 illustrates the flow chart of the present trial, and figure 2 summarises the study protocol.

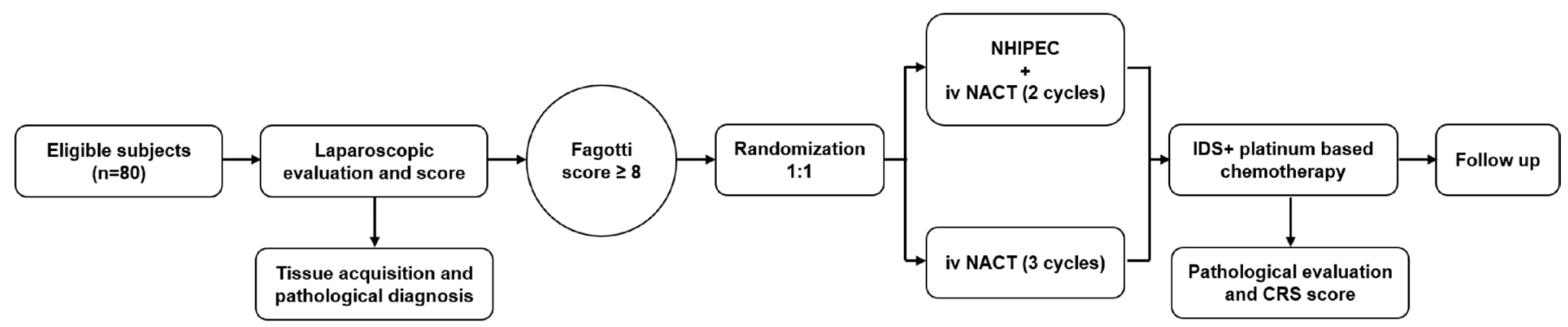

Figure 1 Study design flowchart. CRS, Chemotherapy Response Score; HIPEC, hyperthermic intraperitoneal chemotherapy; IDS, interval debulking surgery; iv NACT, intravenous neoadjuvant chemotherapy; NHIPEC, neoadjuvant HIPEC. 


\begin{tabular}{|c|c|c|c|c|c|}
\hline Time point & Enrollment & Allocation & Neoadjuvant period & Post neoadjuvant period period & Follow-up period \\
\hline \multicolumn{6}{|l|}{ Screening and enrollment } \\
\hline - Inclusion and exclusion criteria & $x$ & & & & \\
\hline - Assessment: & $x$ & & $x$ & $x$ & $x$ \\
\hline \multicolumn{6}{|l|}{ General information } \\
\hline \multicolumn{6}{|l|}{ Medical history collection } \\
\hline \multicolumn{6}{|l|}{ Tumor marker } \\
\hline \multicolumn{6}{|l|}{ Chest and abdominal CT scanning } \\
\hline \multicolumn{6}{|l|}{ Electrocardiogram } \\
\hline \multicolumn{6}{|l|}{ Gynecologic examination } \\
\hline \multicolumn{6}{|l|}{ Physical examination } \\
\hline - Control: iv NACT & & & $x$ & & \\
\hline - IDS & & & & $x$ & \\
\hline - Post-IDS adjuvant chemotherapy & & & & $x$ & \\
\hline \multicolumn{6}{|l|}{ Outcome assessment } \\
\hline - CRS score & & & & $x$ & \\
\hline - AEs & & & $x$ & $x$ & \\
\hline - Survival outcomes evaluation (recurrence and death) & & & $x$ & $x$ & $x$ \\
\hline - Surveillance following the primary treatment & & & & & $x$ \\
\hline
\end{tabular}

Figure 2 Schedule of enrolment, interventions and assessments. AE, adverse event; CRS, Chemotherapy Response Score; IDS, interval debulking surgery; iv NACT, intravenous neoadjuvant chemotherapy; MDT, multidisciplinary team; NACT, neoadjuvant chemotherapy; NHIPEC, neoadjuvant hyperthermic intraperitoneal chemotherapy.

\section{Screening procedure}

Patients who are suspicious for unresectable advanced stage disease can undergo screening for this trial. A preoperative resectability scoring model proposed by Suidan $e t$ $a l,{ }^{29}{ }^{30}$ which is based on CT scan and cancer antigen 125 (CA125), will be used in the screening phase. During the baseline examinations the written informed consent will be obtained from patients with a high risk score $(\geq 7)$ at least 24 hours before laparoscopic evaluation. After the written consent is received, the patient can be registered online.

\section{Endpoints}

Primary endpoint

The proportion of patients who achieve a CRS of 3 following NACT.

Secondary endpoints

1. PFS.

2. OS.

3. Rate of $\mathrm{R} 0$ resection.

4. NHIPEC-related AEs.

\section{Patient eligibility}

Inclusion criteria

1. International Federation of Gynecology and Obstetrics stage IIIC-IVA, HGSOC.

2. Age $18-70$ years.
3. Patients with Fagotti score $\geq 8,{ }^{31}$ suggesting that these patients have a low likelihood to achieve $\mathrm{R} 0$ resection in PDS.

4. Adequate kidney function (blood creatinine 58$96 \mu \mathrm{mol} / \mathrm{L})$.

5. Adequate haematological function (haemoglobin $\geq 110 \mathrm{~g} / \mathrm{L}$, leucocytes $\geq 4.0 \times 10^{9} / \mathrm{L}$, neutrophils $\geq 2.0 \times 10^{9} / \mathrm{L}$, platelets $\geq 100 \times 10^{9} / \mathrm{L}$ ) .

6. Adequate liver function (serum total bilirubin 3.4-22.2 $\mu \mathrm{mol} / \mathrm{L}$, alanine aminotransferase (ALT) 7-40 U/L, aspartate aminotransferase (AST) 13$35 \mathrm{U} / \mathrm{L}, \mathrm{AST} / \mathrm{ALT} \leq 1.5)$.

7. WHO performance status score (WHO score) 0-2.

Exclusion criteria

1. Patients who had received chemotherapy, radiotherapy or any kind of targeted therapy.

2. Patients with complete intestine obstruction.

3. Expected life span $\leq 8$ weeks.

4. Complicated with any other known malignancies.

5. Patients with poor cardiopulmonary function, which would limit compliance with study requirements.

Withdrawal criteria

1. Allergy to platinum compounds or taxanes.

2. Withdrawal of informed consent.

3 . Inability to comply with the protocol or study procedures. 
Table 1 Fagotti scoring algorithm

\begin{tabular}{|c|c|c|}
\hline $\begin{array}{l}\text { Tumour } \\
\text { characteristics }\end{array}$ & Score 0 & Score 2 \\
\hline $\begin{array}{l}\text { Peritoneal } \\
\text { carcinomatosis }\end{array}$ & $\begin{array}{l}\text { Carcinomatosis involving a limited area (along } \\
\text { the paracolic gutter or the pelvic peritoneum) and } \\
\text { surgically removable by peritonectomy }\end{array}$ & $\begin{array}{l}\text { Unresectable massive peritoneal involvement with } \\
\text { a miliary pattern of distribution }\end{array}$ \\
\hline $\begin{array}{l}\text { Diaphragmatic } \\
\text { involvement }\end{array}$ & $\begin{array}{l}\text { No infiltrating carcinomatosis and no nodules } \\
\text { confluent with most of the diaphragmatic surface }\end{array}$ & $\begin{array}{l}\text { Widespread infiltrating carcinomatosis or nodules } \\
\text { confluent with most of the diaphragmatic surface }\end{array}$ \\
\hline $\begin{array}{l}\text { Omental } \\
\text { involvement }\end{array}$ & $\begin{array}{l}\text { No tumour diffusion observed along the omentum up } \\
\text { to the greater curvature of the stomach }\end{array}$ & $\begin{array}{l}\text { Tumour diffusion observed along the omentum up } \\
\text { to the greater curvature of the stomach }\end{array}$ \\
\hline Bowel infiltration & $\begin{array}{l}\text { No bowel resection assumed and no miliary } \\
\text { carcinomatosis observed on the bowel ansae }\end{array}$ & $\begin{array}{l}\text { Bowel resection assumed or miliary } \\
\text { carcinomatosis observed on the ansae }\end{array}$ \\
\hline
\end{tabular}

A value of 0 or 2 is assigned depending on whether disease is present in these locations. If patients score $\geq 8$, optimal cytoreduction is very unlikely. If they score $<8$, they are considered candidates for cytoreductive surgery

4. Use of concomitant medication, including chemotherapeutic drugs and targeted anticancer drugs, or radiotherapy.

\section{Sample size calculation}

The primary endpoint is to compare the CRS 3 rates of the two arms. A previous study reported that CRS 3 could be achieved in $30 \%$ of patients with HGSOC following traditional intravenous NACT. ${ }^{25}$ Based on our previous study, ${ }^{32}$ we hypothesised that a combination of NHIPEC and intravenous NACT will increase the CRS 3 rate by $15 \%$. Using these rates in Simon's selection design, ${ }^{33} 35$ subjects per treatment arm are needed to allow for the selection of the superior treatment arm with a $90 \%$ probability $(\alpha=0.05)$. To account for a $10 \%$ dropout rate, 5 additional subjects will be recruited to each arm, with an accrual goal of 40 subjects per treatment arm.

\section{Intervention}

\section{Laparoscopic evaluation}

All patients with Suidan scores $\geq 7$ will receive an initial laparoscopic evaluation to take a biopsy to confirm histology and assess resectability. The Fagotti scoring system will be used to determine the possibility of R0 resection in the primary debulking setting (table 1). ${ }^{31}$ Scoring will be performed by two experienced gynaecologic oncologists independently. ${ }^{34}$ Patients with a Fagotti score $\geq 8$ will be offered NACT and subsequent IDS.

\section{NHIPEC}

For patients who are assigned to the experimental group (NHIPEC +intravenous NACT), four tubes will be placed via the laparoscopic ports (two in the bilateral subdiaphragmatic space for use as inlet tubes and two in the pelvic cavity for use as outlet tubes), and docetaxel $\left(60-75 \mathrm{mg} / \mathrm{m}^{2}\right)$ perfusion solution will be infused into the peritoneal cavity through the tubes (Day 0) immediately after the laparoscopic evaluation. Then, perfusate containing cisplatin $\left(75 \mathrm{mg} / \mathrm{m}^{2}\right)$ will be infused 24 hours later (Day 1). According to the anaesthetic protocol for HIPEC, all patients will receive intravenous non-opioid analgesics to relieve pain before cisplatin perfusion is initiated. NHIPEC will be administered at $43^{\circ} \mathrm{C}$ for a duration of $60 \mathrm{~min}$. A high-precision hyperthermic intraperitoneal perfusion treatment system (approved by the State Food and Drug Administration of China, approval No. 2009-3260924), which has a precision of $\pm 0.10^{\circ} \mathrm{C}$ for temperature control and $\pm 5 \%$ for flow control, will be used. Saline solution $(3000 \mathrm{~mL})$ will be used to dissolve the drug, and it will be heated and circulated at a flow rate of $300-500 \mathrm{~mL} / \mathrm{min}$. The perfusion velocity will be adjusted to ensure that the entire abdomen is exposed to the perfusate (the initial velocity will be $300 \mathrm{~mL} / \mathrm{min}$, and then it will be increased gradually until the patient feels bloated or a flow rate of $500 \mathrm{~mL} / \mathrm{min}$ is achieved). A goal intra-abdominal temperature of $43^{\circ} \mathrm{C}$ will be measured by temperature monitoring probes in the infusion and outflow catheters. After NHIPEC treatment (Day 1), the four tubes will be removed immediately to retain as much of the drugs in the abdominal cavity as possible.

\section{Intravenous NACT regimen}

Patients in the experimental group will receive two cycles of intravenous NACT, and the first cycle will be initiated with 4 weeks of NHIPEC. Patients in the control group will receive three cycles of intravenous NACT. The regimen of intravenous NACT is docetaxel $60-75 \mathrm{mg} / \mathrm{m}^{2}$ followed by carboplatin AUC 5 for a 21-day cycle. 
IDS and postsurgical adjuvant chemotherapy

Patients in both arms will undergo IDS within 28-30 days after the last cycle of intravenous NACT. IDS will be carried out in accordance with the National Comprehensive Cancer Network ovarian cancer guidelines. ${ }^{1}$ The extent and complexity of the surgical procedures will be categorised according to the surgical complexity scoring system. ${ }^{35}$ The amount of residual disease following IDS will be classified with the cytoreductive completeness scoring (CCS) system, ${ }^{36}$ where CC-0 (R0 resection) is defined as no visible disease after cytoreduction and CC-1, 2 and 3 (CC-1+) scores (residual tumours less than $2.5 \mathrm{~mm}$, between $2.5 \mathrm{~mm}$ and $2.5 \mathrm{~cm}$ and greater than $2.5 \mathrm{~cm}$, respectively) will be grouped together. All patients will receive at least three cycles of platinum and taxanebased adjuvant chemotherapy. ${ }^{1237}$

\section{CRS scoring system}

Prior to the initiation of this trial, all pathologists involved in the present trial will be required to review the original publication by Böhm et $a l^{12}$ and they will receive online training for the CRS system (www.gpecimage.ubc.ca/ aperio/images/crs).

All tissue samples harvested during IDS will be subjected to H\&E expert pathological evaluation to confirm the diagnosis of HGSOC. Omental slides will be independently reviewed by two pathologists to determine those with the greatest amount of viable tumour, and one slide of each site will be selected. The two pathologists, who will be blinded to the written report and each other's results, will independently score each slide according to the CRS system (table 2) ${ }^{12}$ All cases in which the score is not unanimous will be reviewed jointly with a multiheaded microscope. The agreed on consensus score will be used for the final analysis.

\section{Randomisation}

After laparoscopic evaluation is completed, all eligible patients will be randomised in a 1:1 ratio to receive the experimental treatment or the control treatment. The random allocation sequence will be generated using SAS statistical software V.9.4 (SAS Institute) by a biostatistician who works in the Clinical Research Design Division at Sun Yat-sen Memorial Hospital and is not involved in the trial. Then, the sequence will be sealed in an opaque envelope and sent to the investigator.

\section{Monitoring}

The Data and Safety Monitoring Committee (DSMC) will be established, which consists of independent experts who have no conflicts of interest and who agree with the study protocol. The DSMC is responsible for reviewing the study progress, checking the original data on efficacy endpoints and monitoring the safety data. No interim analysis is planned for this trial.

\section{Assessment of safety outcomes}

Safety outcomes include AEs during primary treatment, HIPEC-related AEs, patient-reported AEs and suspected
Table 2 Criteria for the Chemotherapy Response Score (CRS)

CRS1 No or minimal tumour response. Mainly viable tumour with no or minimal regression-associated fibroinflammatory changes, limited to a few foci; cases in which it is difficult to decide between regression and tumour-associated desmoplasia or inflammatory cell infiltration.

\begin{tabular}{|c|c|}
\hline CRS2 & $\begin{array}{l}\text { Appreciable tumour response amid viable tumour } \\
\text { that is readily identifiable. Tumour is regularly } \\
\text { distributed, ranging from multifocal or diffuse } \\
\text { regression-associated fibroinflammatory changes } \\
\text { with viable tumour in sheets, streaks or nodules to } \\
\text { extensive regression-associated fibroinflammatory } \\
\text { changes with multifocal residual tumour, which is } \\
\text { easily identifiable. }\end{array}$ \\
\hline CRS3 & $\begin{array}{l}\text { Complete or near-complete response with no } \\
\text { residual tumour OR minimal irregularly scattered } \\
\text { tumour foci seen as individual cells, cell groups } \\
\text { or nodules up to } 2 \mathrm{~mm} \text { maximum size. Mainly } \\
\text { regression-associated fibroinflammatory changes } \\
\text { or, in rare cases no or very little residual tumour } \\
\text { in the complete absence of any inflammatory } \\
\text { response. It is advisable to record whether } \\
\text { there is no residual tumour or whether there is } \\
\text { microscopic residual tumour present. }\end{array}$ \\
\hline
\end{tabular}

Regression-associated fibroinflammatory changes consist of fibrosis associated with macrophages, including foam cells, mixed inflammatory cells and psammoma bodies, as distinguished from tumour-related inflammation or desmoplasia.

unexpected serious adverse reactions (SUSARs). All AEs occurring during primary treatment will be graded by the National Cancer Institute Common Terminology Criteria for Adverse Events (CTCAE) V.5.0. ${ }^{38}$ Patient-reported AEs will be assessed using a patient-reported version of CTCAE (PRO-CTCAE). ${ }^{39}$ AEs that occur within 3 weeks of NHIPEC are defined as NHIPEC-related. If any serious NHIPEC-related AEs and SUSARs occur during the trial, the investigator will immediately provide appropriate diagnosis and treatment and will report the incidence to the principal investigator and DSMC within 24 hours.

\section{Follow-up}

The follow-up schedule is detailed in box 1 .

\section{Data collection, data management and monitoring}

The data will be stored and handled while maintaining the anonymity of the participants. All data will be collected in a dedicated paper file and reported by the principal investigator or his delegated representative using the EpiData system. All study documents will be regarded as confidential. Once the data are checked, they will be fixed by the DSMC.

\section{Statistical analysis}

Recruitment start date is 1 October 2021 and the planned recruitment end date is 1 October 2022. The results of the present trial are expected to be presented in 2024. 


\section{Box 1 Follow-up schedule}

During the first 2 years of completion of adjuvant chemotherapy

- The follow-up is scheduled every 3 months.

- History is to be obtained with special emphasis on treatment-related morbidity.

- Gynaecological examination.

- Physical examination.

- Cancer antigen 125 (CA125), human epididymis protein 4 (HE4).

- Vaginal ultrasound*.

- CT scan: 3, 6, 12, 18 and 24 months following the completion of adjuvant chemotherapy.

3-5 years following the completion of adjuvant chemotherapy

- The follow-up is scheduled every 6 months.

- History is to be obtained with special emphasis on treatment-related morbidity.

Gynaecological examination.

Physical examination.

- CA125, HE4.

- Vaginal ultrasound*

- CT scan.

${ }^{*}$ Optional

The statistical analysis will be performed by an independent biostatistician. This trial includes four data sets for analysis: intention-to-treat (ITT), per-protocol set (PPS), full analysis set (FAS), and safety set (SAS). The ITT analysis will include all participants, while the PPS analysis will only include participants who strictly follow the protocol and finish the trial. The FAS analysis will include all randomised participants within the respective treatment group they have been assigned to at randomisation according to the ITT principle. The SAS analysis will be carried out to analyse safety outcomes for all randomised participants who receive at least one cycle of neoadjuvant treatment.

Continuous variables will be tested for normality by using the Kolmogorov-Smirnov test. Student's $t$-test will be used to compare normally distributed continuous variables, while the Mann-Whitney $U$ test will be used for data with non-normal distributions. The $\chi^{2}$ test or Fisher's exact test will be used to analyse the frequency distributions of categorical variables where appropriate. PFS and OS will be measured from the date of randomisation until the date of events (recurrence OR death of any cause). PFS and OS will be estimated using the KaplanMeier method and compared with the log-rank test. If the proportional hazards assumption is not violated, Cox proportional hazards models will be fit to estimate the treatment HR and corresponding 95\% CI. All statistical tests will be two-sided, with $\mathrm{p}<0.05$ considered significant. The statistical analysis will be carried out using SAS V.9.4 (SAS Institute).

\section{PATIENT AND PUBLIC INVOLVEMENT}

Patients were not directly involved in the development of the study protocol. Results of the trial will be disseminated to participants through direct consultation with a trial clinician when the trial is completed.

\section{ETHICS AND DISSEMINATION}

The trial will be conducted in accordance with the seventh revision of the Declaration of Helsinki 2013. The study protocol was reviewed and approved by the Institutional Review Board of Sun Yat-sen Memorial Hospital (No. 2020-KY-050). The study was registered on 12 September 2020 with http://www.chictr.org.cn/. The investigators will obtain written informed consent from each participant before screening. Results from this trial will be submitted for publication to peer-reviewed journals, and to national meetings in presentation form.

\section{DISCUSSION}

Although NACT-IDS is recommended in many clinical guidelines for selected patients with ovarian cancer with advanced disease, ${ }^{12}$ its benefit has been a source of longstanding controversy. ${ }^{40}$ Level I evidence supporting this treatment comes from the European Organization for Research and Treatment of Cancer (EORTC) 55971 trial, the Chemotherapy OR Upfront Surgery (CHORUS) trial and the Surgical Complications Related to Primary or Interval Debulking in Ovarian Neoplasm (SCORPION) trial, ${ }^{8-10}$ where similar PFS and OS rates were noted between the NACT-IDS group and the PDS group. In addition, patients treated with NACT were noted to have a lower incidence of postoperative complications and a higher likelihood of achieving R0 cytoreduction than those treated with PDS. However, the non-inferiority of NACT with regard to survival outcomes was not confirmed in the Japan Clinical Oncology Group (JCOG) 0206 trial. $^{41}$ In addition, increasing evidence indicates that the use of NACT is associated with an increased risk of platinum-resistant recurrence. ${ }^{42}$ Collectively, there is an unmet clinical need for a new method to improve the efficacy of traditional intravenous NACT.

In ovarian carcinoma, the disease is primarily confined to the peritoneal cavity. Intraperitoneal administration improves drug delivery to the peritoneal surface, while hyperthermia is directly cytotoxic, activates heat-shock proteins, induces apoptosis, inhibits angiogenesis, promotes protein denaturation, increases the penetration of chemotherapy at the peritoneal surface and increases the chemosensitivity of cancer cells. ${ }^{22}{ }^{43-45}$ Based on the evidence, we proposed NHIPEC in 2019. ${ }^{21}$ Since NHIPEC is delivered using a closed technique, it allows for an increased intra-abdominal pressure and thus improves drug penetration. ${ }^{46}$ Recently, we conducted a multicentre retrospective cohort study and found that the addition of NHIPEC was associated with an increased rate of CRS $3{ }^{32}$ However, the study is limited by limited sample size and selection bias arising from the retrospective design. In the present randomised controlled trial, we aimed to investigate whether the combination of NHIPEC and 
intravenous NACT could show superior efficacy to intravenous NACT alone. To the best of our knowledge, this trial is the first randomised controlled trial to evaluate the effect of HIPEC in the neoadjuvant setting.

The CRS system is used as the primary outcome measure, which we believe is the most notable feature of the NHIPEC trial. Since its description in $2015,{ }^{12}$ the CRS system has been evaluated in many studies, and its prognostic value and reproducibility have been validated. ${ }^{13}{ }^{24-27}$ Based on the evidence, the ICCR guideline has recommended the use of the CRS to assess the histological effects of NACT on patients with HGSOC to enable standardised and objective reporting. ${ }^{28}$ In addition, the Society of Gynecologic Oncology (SGO) White Paper on an The Food and Drug Administration (FDA) Ovarian Cancer Clinical Trial Endpoints Workshop in 2015 highlighted the potential of NACT response to act as a platform for the regulatory approval of novel therapies. ${ }^{47}$ CRS 3, which indicates improved PFS and OS, is a reliable biomarker for patients with HGSOC in the neoadjuvant setting. ${ }^{25}$ Therefore, we believe the significance of the CRS system is in line with what is highlighted in the SGO White Paper. ${ }^{47}$ Currently, ACTRN12618000109202 and KGOG3046 have incorporated the CRS system as an endpoint, which can facilitate timely evaluation of the efficacy of neoadjuvant treatment before IDS.

In van Driel's trial, patients in both groups received three cycles of intravenous chemotherapy following IDS. ${ }^{19}$ The addition of HIPEC to IDS resulted in better survival outcomes than surgery alone. However, it remains unclear whether the beneficial effect is a result of one additional cycle of intraperitoneal chemotherapy. Previous studies have reported that intraperitoneal cisplatin is absorbed into the circulation in the HIPEC setting. In Cashin's study, ${ }^{48}$ a combination of cisplatin $\left(50 \mathrm{mg} / \mathrm{m}^{2}\right)$ and doxorubicin $\left(15 \mathrm{mg} / \mathrm{m}^{2}\right)$ was added to the perfusate and infused for a duration of $90 \mathrm{~min}$. The reported mean half-life (t1/2) of perfusate cisplatin was $18.4 \mathrm{~min}$. The authors concluded that after $75 \mathrm{~min}$, there is little active cisplatin left in the perfusate. Data from our previous study also indicated efficient uptake of cisplatin during HIPEC; approximately $80 \%$ of cisplatin could be used after 60 min of HIPEC. ${ }^{49}$ In the present trial, we sought to investigate whether patients benefit more from a combination of NHIPEC and intravenous NACT than intravenous NACT alone when the same number of cycles of neoadjuvant treatment is prescribed. Therefore, we chose to administer three cycles of intravenous NACT in the control arm and two cycles of intravenous NACT in the experimental arm. In addition, since docetaxel is reported to be effective for peritoneal metastases due to higher concentration and augmentation by heat, ${ }^{50}$ a combination of docetaxel and cisplatin is used for NHIPEC.

A possible limitation of the current trial is interoperator variations in terms of operational technique. To minimise the risk of bias resulted from these variations, only one experienced surgical oncologists specialised in gynaecological malignancies will be required to perform debulking surgery.

In summary, the NHIPEC trial will clarify whether a combination of NHIPEC and intravenous NACT could offer more benefit to patients with HGSOC than intravenous NACT alone. We expect that the addition of NHIPEC will contribute to the efficacy improvement of NACT.

\section{Author affiliations}

${ }^{1}$ Department of Gynecologic Oncology, Sun Yat-sen Memorial Hospital, Guangzhou, Guangdong, China

${ }^{2}$ Clinical Research Design Division, Sun Yat-sen Memorial Hospital, Guangzhou,

Guangdong, China

${ }^{3}$ Department of Clinical Research, Sun Yat-sen University Cancer Center, Guangzhou, China

${ }^{4}$ Radiation Oncology, Dana-Farber Cancer Institute, Boston, Massachusetts, USA

Contributors MFW, ZQL and JL contributed to the conception and design of this work. MFW, LJW, CHL, HWL, TTY, BZZ, QC, YPP and HZ contributed to data collection. YFY and JBL provided statistical expertise. JL prepared the first draft of the manuscript. MFW, YFY and JBL contributed to the analysis and interpretation of the data. All authors provided critical revisions and approved the final version.

Funding The authors have not declared a specific grant for this research from any funding agency in the public, commercial or not-for-profit sectors.

Competing interests None declared.

Patient and public involvement Patients and/or the public were not involved in the design, or conduct, or reporting, or dissemination plans of this research.

Patient consent for publication Not applicable.

Provenance and peer review Not commissioned; externally peer reviewed.

Open access This is an open access article distributed in accordance with the Creative Commons Attribution Non Commercial (CC BY-NC 4.0) license, which permits others to distribute, remix, adapt, build upon this work non-commercially, and license their derivative works on different terms, provided the original work is properly cited, appropriate credit is given, any changes made indicated, and the use is non-commercial. See: http://creativecommons.org/licenses/by-nc/4.0/.

\section{ORCID iDs}

Ji-Bin Li http://orcid.org/0000-0001-7632-3648

Jing Li http://orcid.org/0000-0003-1361-7790

\section{REFERENCES}

1 Armstrong DK, Alvarez RD, Bakkum-Gamez JN, et al. NCCN guidelines insights: ovarian cancer, version 1.2019. J Natl Compr Canc Netw 2019;17:896-909.

2 Colombo N, Sessa C, du Bois A, et al. ESMO-ESGO consensus conference recommendations on ovarian cancer: pathology and molecular biology, early and advanced stages, borderline tumours and recurrent disease. Ann Oncol 2019;30:672-705.

3 Filippova OT, Chi DS, MSKCC Team Ovary. The Unyielding fight against the premature death of patients with advanced-stage ovarian cancer. Obstet Gynecol 2018;132:541-4.

4 Winter WE, Maxwell GL, Tian C, et al. Tumor residual after surgical cytoreduction in prediction of clinical outcome in stage IV epithelial ovarian cancer: a gynecologic Oncology Group study. J Clin Oncol 2008;26:83-9.

5 Benedetti Panici P, Di Donato V, Fischetti M, et al. Predictors of postoperative morbidity after cytoreduction for advanced ovarian cancer: analysis and management of complications in upper abdominal surgery. Gynecol Oncol 2015;137:406-11.

6 Kumar A, Janco JM, Mariani A, et al. Risk-prediction model of severe postoperative complications after primary debulking surgery for advanced ovarian cancer. Gynecol Oncol 2016;140:15-21.

7 Horowitz NS, Miller A, Rungruang B, et al. Does aggressive surgery improve outcomes? Interaction between preoperative disease burden and complex surgery in patients with advanced-stage ovarian cancer: an analysis of GOG 182. J Clin Oncol 2015;33:937-43. 
8 Vergote I, Tropé CG, Amant F, et al. Neoadjuvant chemotherapy or primary surgery in stage IIIC or IV ovarian cancer. N Engl J Med 2010;363:943-53.

9 Kehoe S, Hook J, Nankivell M, et al. Primary chemotherapy versus primary surgery for newly diagnosed advanced ovarian cancer (CHORUS): an open-label, randomised, controlled, non-inferiority trial. Lancet 2015;386:249-57.

10 Fagotti A, Ferrandina MG, Vizzielli G, et al. Randomized trial of primary debulking surgery versus neoadjuvant chemotherapy for advanced epithelial ovarian cancer (SCORPION-NCT01461850). Int J Gynecol Cancer 2020;30:1657-64.

11 Bristow RE, Tomacruz RS, Armstrong DK, et al. Survival effect of maximal cytoreductive surgery for advanced ovarian carcinoma during the platinum era: a meta-analysis. $J$ Clin Oncol 2002;20:1248-59.

12 Böhm S, Faruqi A, Said I, et al. Chemotherapy response score: development and validation of a system to quantify histopathologic response to neoadjuvant chemotherapy in tubo-ovarian high-grade serous carcinoma. J Clin Oncol 2015;33:2457-63.

13 Zorzato PC, Zannoni GF, Tudisco R, et al. External validation of a 'response score' after neoadjuvant chemotherapy in patients with high-grade serous ovarian carcinoma with complete clinical response. Int J Gynecol Cancer 2020;30:67-73.

14 Ditzel HM, Strickland KC, Meserve EE, et al. Assessment of a chemotherapy response score (CRS) system for tubo-ovarian high-grade serous carcinoma (HGSC). Int J Gynecol Pathol 2019;38:230-40.

15 Liang W-F, Li H, Wu J-Y, et al. Identification of ovarian cancer patients most likely to achieve chemotherapy response score 3 following neoadjuvant chemotherapy: development of a predictive nomogram. Front Oncol 2020;10:560888.

16 Blumenthaler AN, Allen CJ, Ikoma N, et al. Laparoscopic HIPEC for low-volume peritoneal metastasis in gastric and gastroesophageal adenocarcinoma. Ann Surg Oncol 2020;27:5047-56.

17 Yang X-J, Huang C-Q, Suo T, et al. Cytoreductive surgery and hyperthermic intraperitoneal chemotherapy improves survival of patients with peritoneal carcinomatosis from gastric cancer: final results of a phase III randomized clinical trial. Ann Surg Oncol 2011;18:1575-81.

18 Yu P, Ye Z, Dai G, et al. Neoadjuvant systemic and hyperthermic intraperitoneal chemotherapy combined with cytoreductive surgery for gastric cancer patients with limited peritoneal metastasis: a prospective cohort study. BMC Cancer 2020;20:1108.

19 van Driel WJ, Koole SN, Sikorska K, et al. Hyperthermic intraperitoneal chemotherapy in ovarian cancer. N Engl J Med 2018;378:230-40.

20 Lei Z, Wang Y, Wang J, et al. Evaluation of cytoreductive surgery with or without hyperthermic intraperitoneal chemotherapy for stage III epithelial ovarian cancer. JAMA Netw Open 2020;3:e2013940.

21 Gao T, Huang X-X, Wang W-Y, et al. Feasibility and safety of neoadjuvant laparoscopic hyperthermic intraperitoneal chemotherapy in patients with advanced stage ovarian cancer: a single-center experience. Cancer Manag Res 2019;11:6931-40.

22 Krawczyk PM, Eppink B, Essers J, et al. Mild hyperthermia inhibits homologous recombination, induces BRCA2 degradation, and sensitizes cancer cells to poly (ADP-ribose) polymerase-1 inhibition. Proc Natl Acad Sci U S A 2011;108:9851-6.

23 Oei AL, Vriend LEM, Crezee J, et al. Effects of hyperthermia on DNA repair pathways: one treatment to inhibit them all. Radiat Oncol 2015; $10: 165$.

24 Santoro A, Angelico G, Piermattei A, et al. Pathological chemotherapy response score in patients affected by high grade serous ovarian carcinoma: the prognostic role of omental and ovarian residual disease. Front Oncol 2019;9:778.

25 Cohen PA, Powell A, Böhm S, et al. Pathological chemotherapy response score is prognostic in tubo-ovarian high-grade serous carcinoma: a systematic review and meta-analysis of individual patient data. Gynecol Oncol 2019;154:441-8.

26 Rajkumar S, Polson A, Nath R, et al. Prognostic implications of histological tumor regression (Böhm's score) in patients receiving neoadjuvant chemotherapy for high grade serous tubal \& ovarian carcinoma. Gynecol Oncol 2018;151:264-8.

27 Lee JY, Chung YS, Na K, et al. External validation of chemotherapy response score system for histopathological assessment of tumor regression after neoadjuvant chemotherapy in tubo-ovarian highgrade serous carcinoma. J Gynecol Oncol 2017;28:e73.

28 McCluggage WG, Judge MJ, Clarke BA, et al. Data set for reporting of ovary, fallopian tube and primary peritoneal carcinoma: recommendations from the International collaboration on cancer reporting (ICCR). Mod Pathol 2015;28:1101-22.
29 Suidan RS, Ramirez PT, Sarasohn DM, et al. A multicenter assessment of the ability of preoperative computed tomography scan and CA-125 to predict gross residual disease at primary debulking for advanced epithelial ovarian cancer. Gynecol Oncol 2017:145:27-31.

30 Kumar A, Sheedy S, Kim B, et al. Models to predict outcomes after primary debulking surgery: independent validation of models to predict suboptimal cytoreduction and gross residual disease. Gynecol Oncol 2019;154:72-6.

31 Fagotti A, Ferrandina G, Fanfani F, et al. A laparoscopy-based score to predict surgical outcome in patients with advanced ovarian carcinoma: a pilot study. Ann Surg Oncol 2006;13:1156-61.

$32 \mathrm{Li} \mathrm{J}$, Wu M-F, Li H, et al. $237 \mathrm{O}$ Effects of neoadjuvant hyperthermic intraperitoneal chemotherapy on chemotherapy response score and recurrence for high-grade serous ovarian cancer patients with advanced disease. Annals of Oncology 2020;31:S1336.

33 Simon R, Wittes RE, Ellenberg SS. Randomized phase II clinical trials. Cancer Treat Rep 1985;69:1375-81.

34 Nick AM, Coleman RL, Ramirez PT, et al. A framework for a personalized surgical approach to ovarian cancer. Nat Rev Clin Oncol 2015:12:239-45.

35 Aletti GD, Dowdy SC, Podratz KC, et al. Relationship among surgical complexity, short-term morbidity, and overall survival in primary surgery for advanced ovarian cancer. Am J Obstet Gynecol 2007;197:676.e1-676.e7.

36 Hoskins WJ, McGuire WP, Brady MF, et al. The effect of diameter of largest residual disease on survival after primary cytoreductive surgery in patients with suboptimal residual epithelial ovarian carcinoma. Am J Obstet Gynecol 1994;170:974-80.

37 Berek JS, Kehoe ST, Kumar L, et al. Cancer of the ovary, fallopian tube, and peritoneum. Int J Gynaecol Obstet 2018;143:59-78.

38 Freites-Martinez A, Santana N, Arias-Santiago S, et al. Using the Common Terminology Criteria for Adverse Events (CTCAE - Version 5.0) to Evaluate the Severity of Adverse Events of Anticancer Therapies. Actas Dermosifiliogr 2021;112:90-2.

39 Dueck AC, Mendoza TR, Mitchell SA, et al. Validity and reliability of the US National cancer Institute's patient-reported outcomes version of the common terminology criteria for adverse events (PROCTCAE). JAMA Oncol 2015;1:1051-9.

40 Long Roche K, Chi DS. Practical guidelines for triage to neoadjuvant chemotherapy in advanced ovarian cancer: big risk, big reward...or too much risk? Gynecol Oncol 2020;157:561-2.

41 Onda T, Satoh T, Ogawa G, et al. Comparison of survival between primary debulking surgery and neoadjuvant chemotherapy for stage III/IV ovarian, tubal and peritoneal cancers in phase III randomised trial. Eur J Cancer 2020;130:114-25.

42 Luo Y, Lee M, Kim HS, et al. Effect of neoadjuvant chemotherapy on platinum resistance in stage IIIC and IV epithelial ovarian cancer. Medicine 2016;95:e4797.

43 van de Vaart PJ, van der Vange N, Zoetmulder FA, et al Intraperitoneal cisplatin with regional hyperthermia in advanced ovarian cancer: pharmacokinetics and cisplatin-DNA adduct formation in patients and ovarian cancer cell lines. Eur $J$ Cancer 1998;34:148-54

44 Panteix G, Beaujard A, Garbit F, et al. Population pharmacokinetics of cisplatin in patients with advanced ovarian cancer during intraperitoneal hyperthermia chemotherapy. Anticancer Res 2002;22:1329-36.

45 Ohno S, Siddik ZH, Kido Y, et al. Thermal enhancement of drug uptake and DNA adducts as a possible mechanism for the effect of sequencing hyperthermia on cisplatin-induced cytotoxicity in L1210 cells. Cancer Chemother Pharmacol 1994;34:302-6.

46 Goldenshluger M, Zippel D, Ben-Yaacov A, et al. Core body temperature but not intraabdominal pressure predicts postoperative complications following Closed-System hyperthermic intraperitonea chemotherapy (HIPEC) administration. Ann Surg Oncol 2018;25:660-6.

47 Herzog TJ, Ison G, Alvarez RD, et al. FDA ovarian cancer clinical trial endpoints workshop: a Society of gynecologic oncology white paper. Gynecol Oncol 2017;147:3-10.

48 Cashin $\mathrm{PH}$, Ehrsson H, Wallin I, et al. Pharmacokinetics of cisplatin during hyperthermic intraperitoneal treatment of peritoneal carcinomatosis. Eur J Clin Pharmacol 2013;69:533-40.

49 Wang W-Y, Wu M-F, Wu D-B, et al. Calculating the dose of cisplatin that is actually utilized in hyperthermic intraperitonea chemotherapy among ovarian cancer patients. J Ovarian Res 2021;14:9.

50 Brandl A, Prabhu A. Intraperitoneal chemotherapy in the treatment of gastric cancer peritoneal metastases: an overview of common therapeutic regimens. J Gastrointest Oncol 2021;12:S32-44. 